\title{
Фотоэлектромагнитный эффект, индуцированный терагерцовым излучением, в топологических изоляторах $\left(\mathrm{Bi}_{1-x} \mathrm{Sb}_{x}\right)_{2} \mathrm{Te}_{3}$
}

\author{
() А.В. Галеева ${ }^{1}$, М.А. Гоманько ${ }^{1}$, М.Е. Тамм ${ }^{2}$, Л.В. Яшина ${ }^{2}$, С.Н. Данилов ${ }^{3}$, Л.И. Рябова ${ }^{2}$, Д.Р. Хохлов ${ }^{1,4,9}$ \\ ${ }^{1}$ Московский государственный университет им. М.В. Ломоносова (физический фракультет), \\ 119991 Москва, Россия \\ ${ }^{2}$ Московский государственный университет им. М.В. Ломоносова (химический фракультет), \\ 119991 Москва, Россия \\ ${ }^{3}$ Университет Регенсбурга, \\ D-93053 Регенсбург, Германия \\ ${ }^{4}$ Физический институт им. П.Н. Лебедева Российской академии наук, \\ 119991 Москва, Россия \\ ฯ E-mail: khokhlov@mig.phys.msu.ru
}

(Получена 3 июля 2018 г. Принята к печати 9 июля 2018 г.)

На основании анализа фотоэлектромагнитного эффекта в трехмерных топологических изоляторах $\left(\mathrm{Bi}_{1-x} \mathrm{Sb}_{x}\right)_{2} \mathrm{Te}_{3} \quad(0 \leq x \leq 0.55)$ сделана оценка подвижности поверхностных носителей заряда. Высокая степень вырождения газа носителей в сочетании с низкой энергией возбуждающего терагерцового кванта обеспечивали неравновесный процесс, связанный исключительно с тепловым разогревом носителей. В этих условиях фотоэдс определяется градиентом подвижности поверхностных и объемных носителей. Фотоэдс и, следовательно, градиент подвижности полностью исчезают при увеличении объемной подвижности до $10^{5} \mathrm{~cm}^{2} \cdot \mathrm{B}^{-1} \cdot \mathrm{c}^{-1}$. В образцах со сравнительно низкой объемной подвижностью при тех же условиях эксперимента фотоэдс отчетливо наблюдается.

DOI: $10.21883 /$ FTP.2019.01.46984.8949

\section{1. Введение}

Энергетический спектр узкозонных полупроводников $\mathrm{Bi}_{2} \mathrm{Te}_{3}, \mathrm{Sb}_{2} \mathrm{Te}_{3}$ и их твердых растворов $\left(\mathrm{Bi}_{1-x} \mathrm{Sb}_{x}\right)_{2} \mathrm{Te}_{3}$ имеет инверсную структуру термов, формирующих зону проводимости и валентную зону $[1,2]$. Согласно теоретическим представлениям, на поверхности этих соединений присутствует топологический слой, характеризующийся линейным законом дисперсии и спиновой поляризацией носителей $[3,4]$. Этот факт экспериментально подтвержден при исследовании фотоэлектронных спектров (ARPES) [5,6].

Для практических приложений приоритетной задачей можно считать определение возможного влияния топологических состояний на транспортные процессы. Однако подобные исследования существенно осложняют высокие значения объемной проводимости, обусловленные сильным вырождением носителей заряда. Успешные попытки снизить концентрацию объемных носителей в этих соединениях являются редким исключением. Согласно имеющимся сведениям, в объемных монокристаллах это было реализовано лишь для одного состава твердых растворов, с $x \approx 0.55$ [7].

Одним из весьма эффективных подходов, позволяющих обнаружить влияние топологического слоя на транспорт носителей при сильном вырождении в объеме, является изучение неравновесных процессов в условиях оптического возбуждения [8-11]. Особый интерес представляют исследования фотоэлектромагнитного (ФЭМ) эффекта, заключающегося в появлении фотоэдс в условиях комбинированного воздействия маг- нитного поля и излучения [12]. При этом важно, что магнитное поле направлено параллельно поверхности образца, излучение направлено нормально поверхности (геометрия Фогта). В частности, в работе [13] было показано, что амплитуда ФЭМ эффекта, индуцированного терагерцовыми (ТГц) лазерными импульсами в кристаллическом топологическом изоляторе $\mathrm{Pb}_{1-x} \mathrm{Sn}_{x} \mathrm{Se}$, определяется диффузией горячих электронов из поверхностного слоя в объем и практически не зависит от объемной концентрации носителей заряда. В дальнейшем в работе [14] при аналогичных исследованиях ФЭМ эффекта в твердых растворах $\left(\mathrm{Bi}_{1-x} \operatorname{In}_{x}\right)_{2} \mathrm{Se}_{3}$, были обнаружены особенности релаксации к равновесному состоянию, присущие только составам в топологической фазе. Различие в характере неравновесных процессов в тривиальной и топологической фазах могут быть связаны со спиновой поляризацией носителей в топологическом слое.

Следует отметить, что в условиях сильного вырождения электронного газа ФЭМ эффект, как правило, обусловлен не генерацией носителей, а их тепловым разогревом [12]. Поскольку ширина запрещенной зоны в $\left(\mathrm{Bi}_{1-x} \mathrm{Sb}_{x}\right)_{2} \mathrm{Te}_{3}$ составляет $\sim 100$ мэВ $[15,16]$, а максимальная энергия кванта излучения не превышает 14 мэВ, межзонная генерация практически исключена.

В работе приведены результаты исследования ФЭМ эффекта для серии образцов, в которых объемная подвижность носителей варьируется в широких пределах. Полученные данные позволили оценить и сравнить подвижности поверхностных и объемных носителей в топологических изоляторах $\left(\mathrm{Bi}_{1-x} \mathrm{Sb}_{x}\right)_{2} \mathrm{Te}_{3}$. 
Гальваномагнитные характеристики образцов при $T=4.2 \mathrm{~K}$

\begin{tabular}{c|l|c|c|c|c|c}
\hline $\begin{array}{c}\text { Номер } \\
\text { образца }\end{array}$ & $x$ & $\begin{array}{c}\text { Тип } \\
\text { проводимости }\end{array}$ & $\begin{array}{c}\rho, \\
\text { мОм } \mathrm{cm}\end{array}$ & $\begin{array}{c}\text { Коэффициент Холла } \\
R_{\mathrm{H}}, \mathrm{cm}^{3} / \text { Кл }\end{array}$ & $\begin{array}{c}\text { Концентрация, } \\
10^{19} \mathrm{~cm}^{-3}\end{array}$ & $\begin{array}{c}\text { Подвижность } \mu, \\
10^{3} \mathrm{~cm}^{2} \cdot \mathrm{B}^{-1} \cdot \mathrm{c}^{-1}\end{array}$ \\
\hline 1 & 0 & $n$ & 0.01 & 0.89 & 0.8 & 110 \\
2 & 0.05 & $p$ & 0.09 & 0.22 & 2.9 & 2.43 \\
3 & 0.1 & $p$ & 0.12 & 0.14 & 4.3 & 1.17 \\
4 & 0.25 & $p$ & 0.19 & 0.14 & 5.3 & 0.76 \\
5 & 0.4 & $p$ & 0.11 & 0.12 & 7.4 & 0.77 \\
6 & 0.55 & $p$ & & 0.09 & & 0.50
\end{tabular}

\section{2. Исследованные образцы и методика эксперимента}

Монокристаллы $\left(\mathrm{Bi}_{1-x} \mathrm{Sb}_{x}\right)_{2} \mathrm{Te}_{3}(0 \leq x \leq 0.55)$ были синтезированы модифицированным методом Бриджмена из шихты стехиометрического состава [17]. Состав синтезированных твердых растворов определяли с помощью рентгенофлюоресцентного анализа.

Для изучения гальваномагнитных свойств от синтезированных слитков откалывались пластины толщиной от 100 до 300 мкм. Образцы имели холловскую конфигурацию. Характерные размеры образца составляли $4 \times 1$ мм. Токовые, потенциальные и холловские контакты нанесены на предварительно напыленные золотые площадки с помощью сплава на основе индия. Измерения проведены в температурном интервале $4.2-300 \mathrm{~K}$ в магнитных полях до 4 Тл. Тип проводимости определялся с помощью термозонда, а также по знаку эдс Холла. Бинарные монокристаллы $\mathrm{Bi}_{2} \mathrm{Te}_{3}$ обладают электронной проводимостью, все твердые растворы $\left(\mathrm{Bi}_{1-x} \mathrm{Sb}_{x}\right)_{2} \mathrm{Te}_{3}-$ дырочной. Вольт-амперные характеристики всех исследованных образцов линейны.

ФЭМ эффект возбуждался терагерцовыми импульсами молекулярного ТГц лазера с оптической накачкой $\mathrm{CO}_{2}$-лазером [18-21]. Длительность импульсов измерялась детекторами на эффекте увлечения [22,23] и составляла $\sim 100$ нс. Длина волны излучения составляла 90 , 148 и 280 мкм, что отвечает значениям энергии кванта $13.8,8.4,4.4$ мэВ соответственно. Падающая мощность достигала 7 кВт и варьировалась с помощью калиброванных аттенюаторов. Пространственное распределение лазерного пучка измерялось пироэлектрической матрицей детекторов $[24,25]$ и соответствовало моде $\mathrm{TEM}_{00}$. Измерения проведены в диапазоне температур $T=4.2-20 \mathrm{~K}$.

\section{3. Экспериментальные результаты}

Значения удельного сопротивления $\rho$, концентрации носителей заряда $p$ и их подвижности $\mu$ (при $T=4.2 \mathrm{~K}$ ) для всех исследованных образцов приведены в таблице. Следует отметить, что в образце с $x=0$ подвижность значительно (на 2 порядка величины) превышает подвижность в твердых растворах, а концентрация свобод-

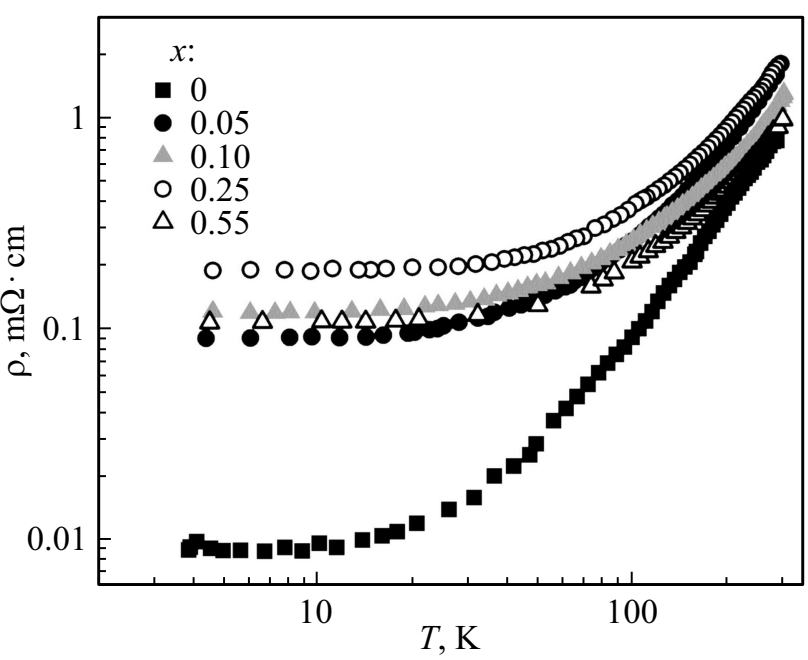

Рис. 1. Температурные зависимости удельного сопротивления образцов $\left(\mathrm{Bi}_{1-x} \mathrm{Sb}_{x}\right)_{2} \mathrm{Te}_{3}$ разного состава.

ных носителей оказывается несколько ниже. Все образцы характеризуются высокой степенью вырождения.

Температурные зависимости удельного сопротивления показаны на рис. 1. Экспериментальные данные для образцов составов с $x=0.4$ и 0.05 практически совпадают, поэтому соответствующие точки для $x=0.4$ не приведены на рисунке. Для всех исследованных образцов в области низких температур, $T \lesssim 10 \mathrm{~K}$, величина $\rho$ практически постоянна. При повышении температуры наблюдается рост сопротивления. Подобные зависимости типичны для вырожденных полупроводников. Их вид определяется температурной зависимостью подвижности, т.е. механизмами рассеяния. В области низких температур это рассеяние на дефектах различного типа, при повышении температуры доминирует рассеяние на тепловых колебаниях решетки.

Фотоэлектромагнитный эффект зарегистрирован во всех исследованных образцах, кроме $\mathrm{Bi}_{2} \mathrm{Te}_{3}$. Для твердых растворов различного состава характерны не только качественные аналогии, но и количественное совпадение основных параметров эффекта. В качестве примера на рис. 2 представлена кинетика эдс ФЭМ эффекта, $U(t)$, для образца с $x=0.05$ в магнитном поле $B=1.5$ Тл при длинах волн возбуждающего излучения $90(a)$, 

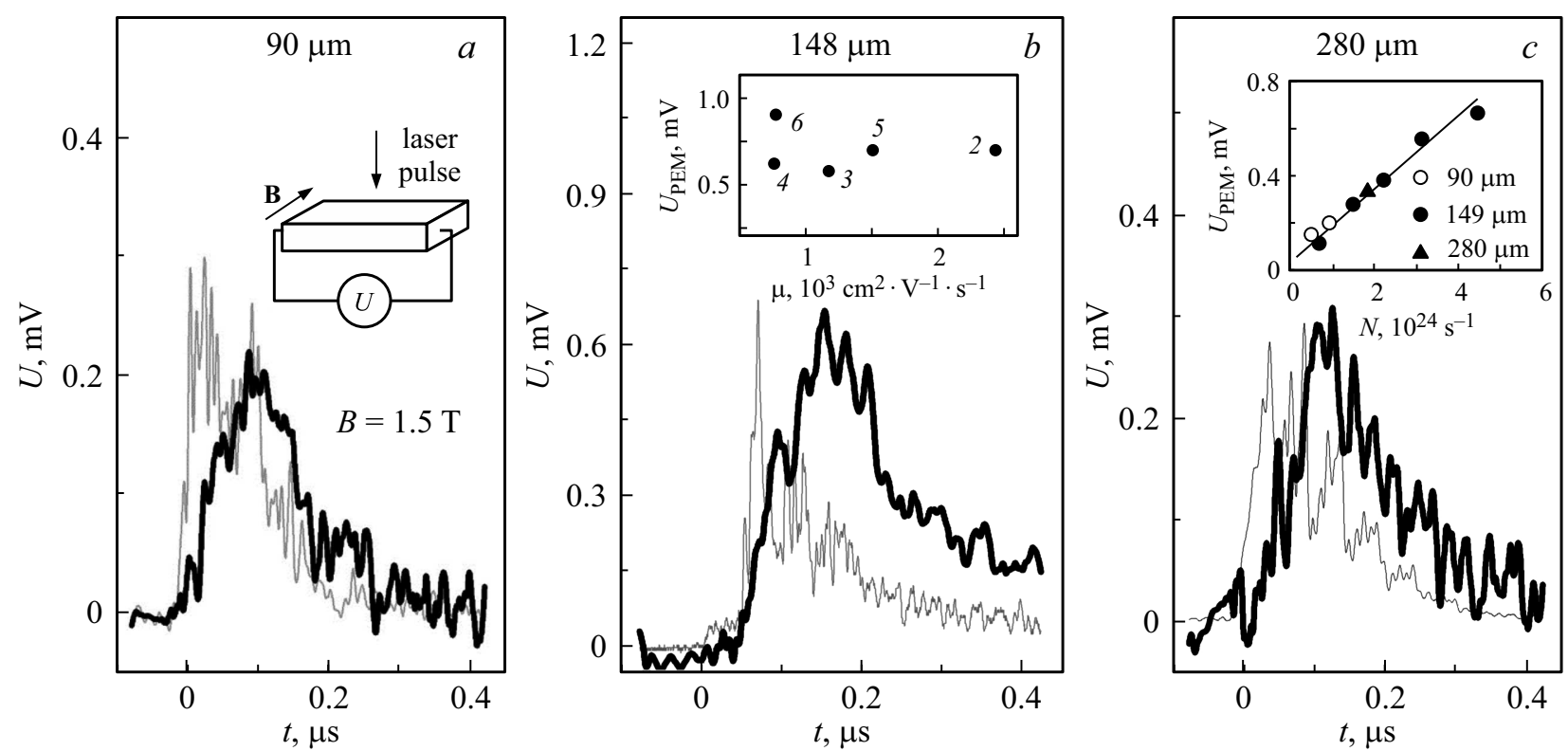

Рис. 2. Кинетика эдс ФЭМ эффекта в образце с $x=0.05$ в магнитном поле $B=1.5$ Тл в условиях возбуждения излучением с длиной волны $90(a), 148(b)$ и 280 мкм $(c)$. Тонкая линия - временной профиль лазерного импульса. На вставках: $a-$ геометрия

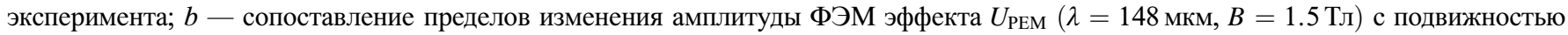

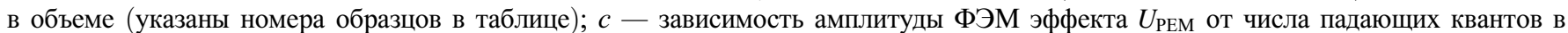
единицу времени для образца состава с $x=0.05$ при $B=1.5$ Тл.

$148(b)$ и 280 мкм $(c)$. Во всех случаях профиль сигнала характеризуется некоторой задержкой по отношению к профилю лазерного импульса. Поскольку импульсы, соответствующие разным длинам волн, имеют разную

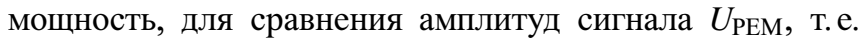
максимального значения регистрируемой эдс, была изучена кинетика ФЭМ эффекта при разной мощности падающего излучения. Измерения проведены в фиксированном магнитном поле $B=1.5$ Тл. Данные, полученные

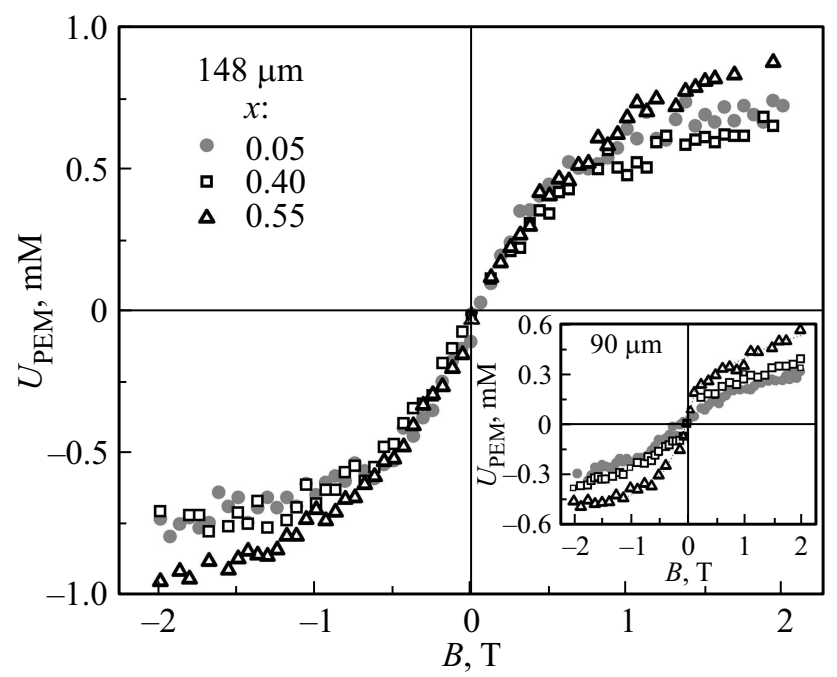

Pис. 3. Зависимость амплитуды ФЭМ эффекта $U_{\mathrm{PEM}}$ от магнитного поля в условиях возбуждения излучением с длиной волны 148 и 90 мкм (на вставке). Составы твердых растворов указаны. в расчете на поток квантов излучения $N$, показаны на вставке к рис. $2, c$. Амплитуда $U_{\mathrm{PEM}}$ линейно зависит от потока квантов $N$ и не зависит от длины волны возбуждающего импульса. Подобная зависимость $U_{\mathrm{PEM}}(N)$ наблюдалась нами ранее в топологических изоляторах на основе селенида висмута [14].

На рис. 3 представлены зависимости амплитуды ФЭМ эффекта $U_{\text {PЕм }}$ от магнитного поля $B$ для составов с $x=0.05,0.4,0.55$ в условиях возбуждения излучением с длиной волны 148 и 90 мкм. Полевые зависимости однотипны и, более того, практически совпадают для всех исследованных образцов. В слабых полях наблюдается практически линейный рост величины $U_{\text {РЕМ, сменяю- }}$ щийся тенденцией к насыщению при увеличении $B$. При смене полярности магнитного поля эффект меняет знак на противоположный.

Дополнительно стоит отметить, что фотоэдс не зависит от поляризации падающего излучения во всех исследованных образцах. При повышении температуры амплитуда ФЭМ эффекта уменьшается, так что фотоэдс не регистрируется уже при $T \approx 15 \mathrm{~K}$. Описанный характер и нечетность $U_{\mathrm{PEM}}(B)$ являются типичными для ФЭМ эффекта в объектах различного типа $[13,14,26]$.

\section{4. Обсуждение результатов}

Принципиальной особенностью ФЭМ эффекта в $\left(\mathrm{Bi}_{1-x} \mathrm{Sb}_{x}\right)_{2} \mathrm{Te}_{3}$ является то, что его индуцирует излучение с энергией кванта, существенно меньшей, чем ширина запрещенной зоны и энергия Ферми. Таким 
образом, и межзонная генерация, и возбуждение с возможных уровней примесей или дефектов в запрещенной зоне практически исключены. Еще одним аргументом, указывающим на отсутствие генерационных процессов, можно считать то, что в идентичных условиях фотопроводимость не наблюдается.

В вырожденных полупроводниках под воздействием излучения с низкими энергиями кванта неравновесное состояние может возникать вследствие теплового разогрева газа носителей. В этом случае эдс ФЭМ эффекта определяется градиентом подвижности в приповерхностном слое $[13,14]$. Знак фотоэдс зависит от направления результирующего потока неравновесных носителей. Важно, что во всех исследованных твердых растворах $\left(\mathrm{Bi}_{1-x} \mathrm{Sb}_{x}\right)_{2} \mathrm{Te}_{3}$ знак фотоэдс соответствует потоку носителей, направленному от поверхности в объем образца. Следовательно, носители на поверхности обладают более высокой подвижностью, чем в объеме.

На рис. $2, b$, на вставке представлены данные, иллюстрирующие пределы изменения амплитуды ФЭМ эффекта $U_{\mathrm{PEM}}$ в зависимости от подвижности объемных носителей $\mu$. В случае изменения подвижности в указанных пределах (в $\sim 3$ раза) корреляции между значениями $U_{\mathrm{PEM}}$ и $\mu$ не прослеживается. Более того, полевые зависимости амплитуды ФЭМ эффекта оказываются практически совпадающими (рис. 3). Это может означать, что отличие объемных электрофизических характеристик образцов с $x>0$ несущественно на фоне значительной разности подвижности в объеме и на поверхности.

В бинарном $\mathrm{Bi}_{2} \mathrm{Te}_{3}$, где ФЭМ эффект не наблюдается, подвижность объемных носителей почти на 2 порядка выше, чем в твердых растворах. Отсутствие фотоэдс, противоположной по знаку, т.е. соответствующей результирующему потоку в направлении к поверхности, указывает на то, что подвижность поверхностных носителей не ниже, чем в объеме. Можно полагать, что в $\mathrm{Bi}_{2} \mathrm{Te}_{3}$ подвижности в объеме и на поверхности оказываются близкими, а результирующий поток возбужденных носителей становится неизмеримо малым. Это позволяет оценить значение подвижности поверхностных носителей в $\mathrm{Bi}_{2} \mathrm{Te}_{3}$ как $\sim 10^{5} \mathrm{~cm}^{2} \cdot \mathrm{B}^{-1} \cdot \mathrm{c}^{-1}$.

Следует отметить, что даже в отсутствие магнитного поля в тонких эпитаксиальных пленках на основе $(\mathrm{Bi}, \mathrm{Sb})_{2} \mathrm{Te}_{3}$ ранее наблюдался фотогальванический эффект, природа которого не связана с диффузией носителей заряда с поверхности в объем образца $[10,11]$. Нечетный по магнитному полю фотогальванический эффект, в частности, может возникать при воздействии терагерцового излучения на орбитальные и спиновые степени свободы электрона в поверхностном слое [27-29]. Однако указанные механизмы, как правило, являются чувствительными к линейной либо циркулярной поляризации падающего излучения. Важно, что подобная чувствительность не обнаружена в настоящей работе. Помимо этого, амплитуда фотогальванического сигнала в двумерном слое $(\mathrm{Bi}, \mathrm{Sb})_{2} \mathrm{Te}_{3}$ слабо зависит от температуры, значительный отклик сохраняется вплоть до комнатной температуры $[10,11]$. В случае же объемного материала, исследованного в настоящей работе, сигнал ФЭМ эффекта пропадает при повышении температуры всего лишь до $15 \mathrm{~K}$. Таким образом, проявление механизмов, наблюдаемых в двумерных системах [27-29], представляется маловероятным. Вполне обоснованно связывать наблюдаемый эффект в исследованных 3D монокристаллах с диффузией неравновесных носителей из приповерхностного слоя.

\section{5. Заключение}

Изучение фотоэдс, возникающей в условиях комбинированного воздействия терагерцового лазерного излучения и магнитного поля, в топологических изоляторах $\left(\mathrm{Bi}_{1-x} \mathrm{Sb}_{x}\right)_{2} \mathrm{Te}_{3}$ позволило обнаружить высокоподвижные поверхностные носители и оценить значение их подвижности. Нельзя исключить, что наличие высокоподвижных носителей в твердых растворах $\left(\mathrm{Bi}_{1-x} \mathrm{Sb}_{x}\right)_{2} \mathrm{Te}_{3}$ может быть обусловлено формированием топологического слоя.

Авторы выражают благодарность проф. С.Д. Ганичеву за ценные замечания.

Работа выполнена при поддержке гранта РНФ № 17-72-10064. С.Н. Данилов благодарит Deutsche Forschungsgemeinschaft (DFG, проект SPP 1666) за поддержку. Экспериментальная инфраструктура в терагерцовом центре Регенсбурга TerZ частично поддержана DAAD программой обмена между МГУ и университетом г. Регенсбург.

\section{Список литературы}

[1] H. Zhang, C.-X. Liu, X.-L. Qi, X. Dai, Z. Fang, S.-C. Zhang. Nature Phys., 5, 438 (2009).

[2] C. Niu, Y. Dai, Y. Zhu, Y. Ma, L. Yu, S. Han, B. Huang. Sci. Rep., 2, 976 (2012).

[3] M.Z. Hasan, C.L. Kane. Rev. Mod. Phys., 82, 3045 (2010).

[4] A. Bansil, H. Lin, T. Das. Rev. Mod. Phys., 88, 021004 (2016).

[5] J. Zhang, C.-Z. Chang, Z. Zhang, J. Wen, X. Feng, K. Li, M. Liu, K. He, L. Wang, X. Chen, Q.-K. Xue, X. Ma, Y. Wang. Nature Commun., 2, 574 (2011).

[6] D. Kong, Y. Chen, J.J. Cha, Q. Zhang, J.G. Analytis, K. Lai, Z. Liu, S.S. Hong, K.J. Koski, S.-K. Mo, Z. Hussain, I.R. Fisher, Z.-X. Shen, Y. Cui. Nature Nanotechnol., 6, 705 (2011).

[7] J. Sánchez-Barriga, M. Battiato, E. Golias, A. Varykhalov, L.V. Yashina, O. Kornilov, O. Rader. Appl. Phys. Lett., 110, 141605 (2017).

[8] R. Valdés Aguilar, A.V. Stier, W. Liu, L.S. Bilbro, D.K. George, N. Bansal, L. Wu, J. Cerne, A.G. Markelz, S. Oh, N.P. Armitage. Phys. Rev. Lett., 108, 087403 (2012).

[9] C.W. Luo, H.-J. Chen, C.M. Tu, C.C. Lee, S.A. Ku, W.Y. Tzeng, T.T. Yeh, M.C. Chiang, H.J. Wang, W.C. Chu, J.-Y. Lin, K.H. Wu, J.Y. Juang, T. Kobayashi, C.-M. Cheng, C.-H. Chen, K.-D. Tsuei, H. Berger, R. Sankar, F.C. Chou, H.D. Yang. Adv. Opt. Mater., 1, 804 (2013). 
[10] H. Plank, J. Pernul, S. Gebert, S.N. Danilov, J. König-Otto, S. Winnerl, M. Lanius, J. Kampmeier, G. Mussler, I. Aguilera, D. Grützmacher, S.D. Ganichev. Phys. Rev. Mat., 2, 024202 (2018).

[11] H. Plank, L.E. Golub, S. Bauer, V.V. Bel'kov, T. Herrmann, P. Olbrich, M. Eschbach, L. Plucinski, C.M. Schneider, J. Kampmeier, M. Lanius, G. Mussler, D. Gruetzmacher, S.D. Ganichev. Phys. Rev. B, 93, 125434 (2016).

[12] И.К. Кикоин, С.Д. Лазарев. УФН, 124, 597 (1978).

[13] S.G. Egorova, V.I. Chernichkin, L.I. Ryabova, E.P. Skipetrov, L.V. Yashina, S.N. Danilov, S.D. Ganichev, D.R. Khokhlov. Sci. Rep., 5, 11540 (2015).

[14] A.V. Galeeva, S.G. Egorova, V.I. Chernichkin, M.E. Tamm, L.V. Yashina, V.V. Rumyantsev, S.V. Morozov, H. Plank, S.N. Danilov, L.I. Ryabova, D.R. Khokhlov. Semicond. Sci. Technol., 31, 095010 (2016).

[15] Д. Лонг. УФН, 80 (8), 639 (1963).

[16] А.Н. Вейс, Л.Н. Лукьянова, В.А. Кутасов. ФТТ, 54 (11), 2051 (2012),

[17] В.И. Штанов. Кристаллография, 49 (2), 343 (2004).

[18] P. Schneider, J. Kainz, S.D. Ganichev, V.V. Bel'kov, S.N. Danilov, M.M. Glazov, L.E. Golub, U. Rössler, W. Wegscheider, D. Weiss, D. Schuh, W. Prettl. J. Appl. Phys., 96, 420 (2004).

[19] V. Lechner, L.E. Golub, P. Olbrich, S. Stachel, D. Schuh, W. Wegscheider, V.V. Bel'kov, S.D. Ganichev. Appl. Phys. Lett., 94, 242109 (2009).

[20] P. Olbrich, J. Karch, E.L. Ivchenko, J. Kamann, B. März, M. Fehrenbacher, D. Weiss, S.D. Ganichev. Phys. Rev. B, 83, 165320 (2011).

[21] P. Olbrich, L.E. Golub, T. Herrmann, S.N. Danilov, H. Plank, V.V. Bel'kov, G. Mussler, Ch. Weyrich, C.M. Schneider, J. Kampmeier, D. Grützmacher, L. Plucinski, M. Eschbach, S.D. Ganichev. Phys. Rev. Lett., 113, 096601 (2014).

[22] С.Д. Ганичев, С.А. Емельянов, И.Д. Ярошецкий. Письма ЖЭТФ, 35, 368 (1982).

[23] С.Д. Ганичев, Я.И. Терентьев, И.Д. Ярошецкий. Письма ЖТФ, 11, 46 (1985).

[24] S.D. Ganichev. Physica B, 273-274, 737 (1999).

[25] E. Ziemann, S.D. Ganichev, I.N. Yassievich, V.I. Perel, W. Prettl. J. Appl. Phys., 87, 3843 (2000).

[26] A.V. Galeeva, I.V. Krylov, K.A. Drozdov, A.F. Knjazev, A.V. Kochura, A.P. Kuzmenko, V.S. Zakhvalinskii, S.N. Danilov, L.I. Ryabova, D.R. Khokhlov. Beilstein J. Nanotechnol., 8, 167 (2017).

[27] V.V. Bel'kov, S.D. Ganichev. Semicond. Sci. Technol., 23, 114003 (2008).

[28] V.V. Bel'kov, S.D. Ganichev, E.L. Ivchenko, S.A. Tarasenko, W. Weber, S. Giglberger, M. Olteanu, H.-P. Tranitz, S.N. Danilov, Petra Schneider, W. Wegscheider, D. Weiss, W. Prettl. J. Phys. C: Condens. Matter, 17, 3405 (2005).

[29] S.D. Ganichev, S.A. Tarasenko, V.V. Bel'kov, P. Olbrich, W. Eder, D.R. Yakovlev, V. Kolkovsky, W. Zaleszczyk, G. Karczewski, T. Wojtowicz, D. Weiss. Phys. Rev. Lett., 102, 156602 (2009).

Редактор Л.В. Шаронова

\section{Photoelectromagnetic effect induced by terahertz radiation in $\left(\mathrm{Bi}_{1-x} \mathbf{S b}_{x}\right)_{2} \mathrm{Te}_{3}$ topological insulators}

\author{
A.V. Galeeva ${ }^{1}$, M.A. Gomanko ${ }^{1}$, M.E. Tamm ${ }^{2}$, \\ L.V. Yashina ${ }^{2}$, S.N. Danilov ${ }^{3}$, L.I. Ryabova ${ }^{2}$, \\ D.R. Khokhlov ${ }^{1,4}$ \\ ${ }^{1}$ Lomonosov Moscow State University \\ (Faculty of Physics), \\ 119991 Moscow, Russia \\ ${ }^{2}$ Lomonosov Moscow State University \\ (Faculty of Chemistry), \\ 119991 Moscow, Russia \\ ${ }^{3}$ Regensburg University, \\ D-93053 Regensburg, Germany \\ ${ }^{4}$ Lebedev Physical Institute, \\ Russian Academy of Sciences, \\ 119991 Moscow, Russia
}

Abstract Our study of the photoelectromagnetic effect excited by the terahertz laser radiation in $\left(\mathrm{Bi}_{1-x} \mathrm{Sb}_{x}\right)_{2} \mathrm{Te}_{3}(0 \leq x \leq 0.55)$ $3 \mathrm{D}$ topological insulators provide us with an estimate of the surface carrier mobility. The non-equilibrium processes originated from the carrier gas heating due to the high degeneracy of the carriers in combination with the low energy of the incident quanta. In these conditions, the voltage induced depends on the difference between the mobility at the sample surface and in the bulk. Whereas the photoelectromagnetic effect is present in the samples with relatively low mobility in the bulk, it is absent in the sample with the bulk mobility as high as $10^{5} \mathrm{~cm}^{2} \cdot \mathrm{V}^{-1} \cdot \mathrm{s}^{-1}$. 